\title{
Increased mean platelet volume in patients with infective endocarditis and embolic events
}

\author{
Mehmet İleri ${ }^{1}$, Selçuk Kanat ${ }^{1}$, Gürdal Orhan ${ }^{2}$, Pınar Türker Bayır ${ }^{1}$, \\ Havva Tuğba Gürsoy ${ }^{1}$, Deniz Şahin ${ }^{1}$, Gökhan Çiçek ${ }^{1}$, Özgül Uçar Elalmış ${ }^{1}$ Ümit Güray ${ }^{1}$ \\ ${ }^{1}$ Department of Cardiology, Ankara Numune Education and Research Hospital, Ankara, Turkey \\ ${ }^{2}$ Department of Neurology, Ankara Numune Education and Research Hospital, Ankara, Turkey
}

\begin{abstract}
Background: Platelet activation appears to play an important role in thromboembolic complications of infective endocarditis (IE). Mean platelet volume (MPV) is a potentially useful marker of platelet activity and a quick and easy determinant of thrombotic risk. Hence the aim of this study was to investigate the baseline platelet volume indices (MPV and platelet distribution width [PDW]) in IE patients who developed embolic events in the follow-up period and who did not.
\end{abstract}

Methods: The study group consisted of 76 consecutive patients (female: 55, male: 21, mean age: 26 years old, ranged: 8-64 years) with definite IE according to Duke Criteria. Thirty four healthy subjects, who were age and gender adjusted, served as the control group. The mean duration of hospital stay was 44 days.

Results: Among the IE patients, 13 (13/76, 17.1\%) had major embolic events. Significantly larger vegetations were observed in patients with embolic events as compared to non-embolic group (1.4 vs. $1.0 \mathrm{~cm}, p=0.03)$. MPV at hospital admission was higher in patients who had embolic events in the follow-up period compared to both those who did not and the control subjects (10.62 \pm 1.13 vs. $9.25 \pm 0.97$ and $8.93 \pm 0.82 \mathrm{fL}, p<0.001$, respectively). Similarly, the patients with embolic events had increased PDW compared to the non-embolic ones and the control group (16.31 \pm 2.42 vs. $14.35 \pm 1.97$ and $14.04 \pm 1.82 \%, p<0.001$, respectively).

Conclusions: The present study demonstrated that IE patients with embolic events had increased MPV and PDW values, compared to non-embolics. Future prospective studies with standardized measurements may clarify the clinical role of platelet volume indices in thromboembolic complications of IE. (Cardiol J 2015; 22, 1: 37-43)

Key words: mean platelet volume, infective endocarditis, thromboembolism

\section{Introduction}

It has long been known that thromboembolic events are common and severe complications of infective endocarditis (IE) which occur in $20 \%$ to $43 \%$ of cases, with the majority as cerebral emboli [1-3].
This wide range of complications points to the heterogeneity of the disease and the critical need for baseline risk stratification in order to focus aggressive management toward a high-risk group of patients. The predictive role of clinical and echocardiographic parameters on the subsequent risk

Address for correspondence: Mehmet İleri, MD, Samur Sokak, 30/10, Kurtuluş, Ankara, Turkey, tel: ++90 5054859803 , e-mail: maraspoli2020@gmail.com

Received: 16.01.2014 Accepted: 13.02.2014 
of embolism was well investigated [4-7]. However, the interrelations between the biomarkers of platelet activity and the risk of embolic events have not been well studied in a large prospective patient population with definite IE. Mean platelet volume (MPV) is a potentially useful marker of platelet activity and a quick and easy determinant of thrombotic risk [8-10]. Platelet distribution width (PDW), another platelet activation marker, shows the variability in platelet size. Hence the aim of this study was to investigate the baseline platelet volume indices (MPV and PDW) in IE patients who developed embolic events in follow up period and who did not.

\section{Methods}

Seventy-six consecutive patients (female: 55 , male: 21 , mean age: 26 years old, ranged: $8-64$ years) admitted to the Department of Cardiology in Türkiye Yüksek İhtisas Hospital with the diagnosis of definite IE according to Duke criteria [11] were included in this study. IE was diagnosed if one of these criteria was present; (1) histopathologic evidence of endocarditis; (2) persistent positive blood cultures, excluding other potential sources of bacteremia, and a new regurgitant murmur or predisposing heart disease; and (3) negative or intermittently positive blood cultures plus fever in the presence of a new regurgitant murmur and microvascular or immunologic phenomena. Exclusion criteria were: patients with a history of thromboembolic events before hospital admittance, patients who had prosthetic heart valves, patients under platelet-inhibitors treatment, patients with hematological disorders or cancer and patients with a cerebral hemorrhage or uncertainty about the diagnosis of an embolic event. Thirty four healthy subjects who were age and gender adjusted (female: 24 , male: 10 , mean age: 24 years old, ranged: 10-63 years) served as the control group. The study protocol was approved by the institutional Ethics Committee and informed consent was given by all patients and control subjects.

The patients were hospitalized and prospectively followed in our clinic until discharge, by a team composed of cardiologists, infectious disease specialists, cardiac surgeons, and neurologists involved in management of the patients. Blood cultures and initial clinical evaluation were systematically performed in all patients at baseline (before the initiation of adequate antibiotic therapy). Antibiotic therapy was started immediately after diagnosis. Blood samples for hematologic variables and platelet volume indices were drawn at 8.00 to 10.00 a.m. hospital admis- sion. Transthoracic and transesophageal echocardiographic examinations were performed within 3 days of initiation of antimicrobial therapy. The mean duration of hospital stay was 44 days. All patients were observed daily by cardiology and infectious disease specialists, and additional consultations were secured when indicated. The diagnosis of an embolic event was based on physical examination, cerebral computed tomography, peripheral Doppler ultrasonographic investigation or angiography. Microvascular emboli such as cutaneous microinfarctions or immune complex phenomena were not regarded as embolus. Thirteen $(17.1 \%)$ patients were diagnosed to develop either a cerebral $(n=7)$, pulmonary $(n=2)$, renal $(n=1)$ or a major peripheral $(n=3$, of which 2 popliteal and 1 femoral artery) embolic event during the in hospital follow-up period. The remaining $63(82.9 \%)$ patients with IE did not have any embolic events.

Transthoracic 2-dimensional and color flow Doppler echocardiography was performed in all patients using ultrasound equipment (Toshiba SSH160A) with commercially available $2.5-\mathrm{MHz}$ transducers. Transesophageal examination was performed within 3 days of initiation of antimicrobial therapy with the use of a $5-\mathrm{MHz}$ phased - array transducer. All echocardiographic studies were recorded on super-VHS videotape for subsequent independent review and analyzed by 2 echocardiographers who were unaware of the clinical status of the patients. In case of disagreement, a third examiner was consulted. The presence and characteristics of vegetations were evaluated according to transesophageal echocardiography. Valvular vegetation was defined as an oscillating or fixed mass associated with a valve or its supporting apparatus, distinct in echogenic structure, and with motion independent from the remainder of the involved leaflet [12]. The lesion had to be detectable throughout the complete cardiac cycle. Diffuse valvular irregularities or valvular thickenings were not regarded as vegetation. Echocardiographic data included the presence, maximal length, mobility and being pedunculated or sessile of vegetation. Measurements of vegetation length were performed in various planes, and maximal length was used. In the presence of multiple vegetations, the largest length was used for analysis. The vegetation was evaluated as immobile when it was fixed with no detectable independent motion or had a fixed base but with a minimal motion of the free edge.

Blood samples at hospital admission were drawn from the antecubital vein by careful venipuncture using a 21-gauge needle attached to 
a sterile syringe at 8.00 to 10.00 a.m. Platelet volume indices were measured in blood samples collected in dipotassium ethylenediaminetetraacetic acid (EDTA) containing tubes by flow cytometry in an automated blood cell counter (Sysmex, XT2000i) immediately within 60 min after sampling. The Sysmex XT 2000i uses the electric resistance detecting method (impedance technology) with hydrodynamic focusing [13]. Hydrodynamic focusing direct current detection is a modification of the optical method and used for the measurement of red blood cell and platete. This method is to enclose the diluted sample ejected from a nozzle with a circumfluent sheath fluid, to allow the sample to flow through the center of an aperture, and to detect the changes in the electric current flowing through the aperture. Fluorescence flow cytometry is used to measure white blood cells (WBC), differential parameters, the optical platelet count and the reticulocyte count. The system employs a $633 \mathrm{~nm}$ semi-conductor laser for flow cytometry analysis. For the measurement by flow cytometry of the proportional count, expressed as percent of the total WBC, of neutrophils, lymphocytes, monocytes and eosinophils, white cells are stained with fluorescent dyes that bind to both DNA and RNA. Side scatter is employed to determine the internal complexity of the cell. Fluorescence and scatter measurements are combined to characterize white cell populations.

\section{Statistical analysis}

Results are expressed as mean \pm standard deviation. Comparison of clinical and echocardiographic variables between patients with and without embolic events was performed using Student's t- test for numerical variables and $\chi^{2}$ test for categorical data. All patients with IE with and embolic events and control subjects were compared for MPV and PDW using the Mann-Whitney U test. A p value $<0.05$ was considered significant.

\section{Results}

The study group consisted of 76 consecutive patients (female: 55 , male: 21 , mean age: 26 years old, ranged: 8-64 years) with definite IE according to Duke Criteria. Patients with a history of thromboembolic events before hospital admittance or those who had prosthetic heart valves, or were being treated with platelet-inhibitors were excluded. Thirty four healthy subjects who were age and gender adjusted (female: 24 , male: 10 , mean age: 24 years old, ranged: $10-63$ years) served as the control group. The mean duration of hospital stay for patients was 44 days.

Among the 76 patients with IE included in the study, $13(17.1 \%)$ developed embolic events (7 cerebral, 2 pulmonary, 1 renal and 3 major peripheral) in follow-up period while the remaining 63 did not have this complication. Thirteen $(17.1 \%)$ patients were diagnosed to develop either a cerebral $(n=7)$, pulmonary $(n=2)$, renal $(n=1)$ or a major periphe$\mathrm{ral}(\mathrm{n}=3$, of which 2 popliteal and 1 femoral artery) embolic event during the in-hospital follow-up period. Clinical and echocardiographic characteristics of patients with and without embolic events are shown in Table 1. Measurements of simple hematological variables and causative microorganisms did not differ significantly between the two groups. Localization, mobility and attachment characteristics of the vegetations were similar between patients with and without embolic complications. Although there was a trend toward a higher number of vegatations detected in those with embolic events, this did not reach statistical significance ( $84.6 \%$ vs. $80.9 \%$, p > 0.05). Significantly larger vegetations were observed in patients with embolic events as compared to the non-embolic group (1.4 vs. $1.0 \mathrm{~cm}, \mathrm{p}=0.03$ ).

MPV at hospital admission was higher in patients who had embolic events in the follow-up period compared to both those who did not and the control subjects $(10.62 \pm 1.13$ vs. $9.25 \pm 0.97$ and $8.93 \pm 0.82 \mathrm{fL}, \mathrm{p}<0.001$, respectively) (Table 2). Similarly, the patients with embolic events had increased PDW compared to non-embolic ones and the control group $(16.31 \pm 2.42$ vs. $14.35 \pm 1.97$ and $14.04 \pm 1.82 \%, \mathrm{p}<0.001$, respectively) (Table 2).

\section{Discussion}

The present study is the first to have a prospective design, comparing the platelet volume indices, namely MPV and PDW, in a relatively large population of IE patients with and without thromboembolic complications. Patients who developed embolic events in follow-up period had significantly increased MPV and PDW values at admission compared to non-embolic ones. Larger platelets in the embolic group reflect enhanced platelet activity and probably identify patients at high thromboembolic risk. These data suggest that MPV is a potentially useful prognostic biomarker for the risk of embolic events in IE patients. Although the precise biological pathways by which elevated MPV might influence the development 
Table 1. Clinical and echocardiographic characteristics of infective endocarditis patients with and without embolic events.

\begin{tabular}{|c|c|c|}
\hline & $\begin{array}{l}\text { Embolic event }(+) \\
\qquad(n=13)\end{array}$ & $\begin{array}{l}\text { Embolic event (-) } \\
\qquad(\mathrm{n}=63)\end{array}$ \\
\hline Age & 28 & 24 \\
\hline Men/women & $4 / 9$ & $17 / 46$ \\
\hline \multicolumn{3}{|l|}{ Hematologic variables: } \\
\hline Hematocrit [\%] & $35.3 \pm 4.1$ & $34.4 \pm 5.2$ \\
\hline Erythrocyte sedimentation rate $[\mathrm{mm} / \mathrm{h}]$ & 98 & 76 \\
\hline Platelet count $\left[\times 10^{9} / \mathrm{L}\right]$ & $265.5 \pm 62.4$ & $245.6 \pm 56.4$ \\
\hline aPTT [s] & $26.8 \pm 4.0$ & $31.5 \pm 6.3$ \\
\hline Fibrinogen [g/L] & $6.1 \pm 1.3$ & $4.2 \pm 1.5$ \\
\hline \multicolumn{3}{|l|}{ Causative microorganisms: } \\
\hline Viridans streptococci & $5(38.4 \%)$ & $22(34.9 \%)$ \\
\hline Group D streptococci and enterococci & $2(15.4 \%)$ & $9(14.2 \%)$ \\
\hline Other streptococci & $1(7.6 \%)$ & $4(6.3 \%)$ \\
\hline Staphylococci & $3(23.1 \%)$ & $14(22 \%)$ \\
\hline Other microorganism & $1(7.6 \%)$ & $7(11.1 \%)$ \\
\hline Undetermined & $1(7.6 \%)$ & $7(11.1 \%)$ \\
\hline Vegetation detected & $11(84.6 \%)$ & $51(80.9 \%)$ \\
\hline \multicolumn{3}{|l|}{ Location of vegetation: } \\
\hline Mitral & $6(54.5 \%)$ & $27(52.9 \%)$ \\
\hline Aortic & $3(27.2 \%)$ & $19(37.2 \%)$ \\
\hline Mitral and aortic & $1(9.1 \%)$ & $3(5.8 \%)$ \\
\hline Tricuspid & $1(9.1 \%)$ & $2(3.9 \%)$ \\
\hline \multicolumn{3}{|l|}{ Vegetation characteristics: } \\
\hline Size $[\mathrm{cm}]$ & $1.4 \pm 0.4^{*}$ & $1.0 \pm 0.3$ \\
\hline Mobile/immobile & $3 / 8$ & $14 / 49$ \\
\hline Pedunculated/sessile & $1 / 10$ & $7 / 56$ \\
\hline
\end{tabular}

${ }^{*} p=0.03$ compared to embolic (-) patients; aPTT — activated partial thromboplastin time

Table 2. Platelet volume indices in infective endocarditis patients with and without embolic events and control subjects.

\begin{tabular}{lccc}
\hline Variables & $\begin{array}{c}\text { Embolic event }(+) \\
(\mathbf{n}=\mathbf{1 3})\end{array}$ & $\begin{array}{c}\text { Embolic event (-) } \\
(\mathbf{n}=\mathbf{6 3})\end{array}$ & $\begin{array}{c}\text { Control group } \\
\text { (n = 34) }\end{array}$ \\
\hline Mean platelet volume [fL] & $10.62 \pm 1.13^{*}$ & $9.25 \pm 0.97$ & $8.93 \pm 0.82$ \\
Platelet distribution width [\%] & $16.31 \pm 2.42^{* *}$ & $14.35 \pm 1.97$ & $14.04 \pm 1.82$ \\
\hline
\end{tabular}

${ }^{*} p<0.001$ compared to embolic event (-) patients and control group; ${ }^{* *} p<0.001$ compared to embolic event (-) patients and control group

of embolic complications, multiple mechanisms may be involved. Larger platelets are metabolically more active and have greater prothrombotic potential with increased thromboxane-A2 and B2 per unit volume and glycoprotein IIb/IIIa receptor expression $[14,15]$. They show more aggregability in response to adenosise diposphate and decreased inhibition of aggregation by prostacyclin in vitro. Larger platelets are denser and contain more alpha granules which can release prothrombotic substances including platelet factor- 4 , $\mathrm{P}$ selectin and platelet-derived growth factor [15].

Some mechanisms suggested to cause systemic embolism in bacterial infections are namely activation of blood coagulation, enhanced platelet activity/damage and endothelial cell injury. Previous investigations demonstrated that systemic bacterial infections, even in the absence of cardiac involvement, represent an independent risk factor for embolic events [16-19]. Inflammation induced 
procoagulant changes and endothelial cell injury favoring thrombosis were suggested as an explanation in this setting. It was reported that, especially severe bloodstream bacterial infections change the prostacyclin-thromboxane ratio in the direction of thrombosis and induce the production of cytokines, interleukin-1 and the tumor necrosis factor, which may lead to injured endothelial cells and increased blood coagulation and platelet aggregation [18, 19]. It is important to note that IE differs from other infectious diseases owing to the presence of cardiac vegetations that represent an additional independent risk factor for embolic events. Previously, we had reported IE patients with subsequent embolizations with increased levels of E-selectin as compared to both those without emboli and healthy subjects [20]. This increase reflects the endothelial dysfunction in these patients, with induction of a pro-adhesive and pro-thrombotic surface causing a subsequent distortion of the endothelial-platelet axis and thrombus formation. Moreover, in the same population, we have found that IE patients with embolic events had significantly higher P-selectin levels, reflecting enhanced platelet activation, which have a direct impact on thrombus formation [20]. The same group of embolic patients also had significantly higher beta-thromboglobulin and platelet factor-4 levels, reflecting enhanced platelet activation/damage [21]. Putting these data together, platelet activation may play an additional role in thromboembolic complications of IE.

Although there has been a wide variety of different methods measuring platelet activity reported, most of them remain a research tool that are yet to be included in routine clinical decision-making. Among these, platelet volume indices are a group of parameters which are inexpensive, routinely measured by automated cell counters and easy to interpret. MPV and PDW are the most validated and prominent of these. As compared to other measures of platelet activity which require special homeostasis laboratories, blood processing procedures, platelet isolation and specialized equipment, MPV is a practical and prognostically important biomarker of platelet reactivity.

Evidence derived from both retrospective and prospective studies suggests that increased MPV is an important determinant of thrombotic risk in predominantly arterial disorders [22-25]. Prospective studies, to a certain extent, support the value of elevated MPV in predicting recurrent myocardial infarction and death in post infarction period [26], impaired myocardial reperfusion after thrombolysis [27] or percutaneous coronary interventions in
ST-elevation myocardial infarction [28], and restenosis after stenting in angina pectoris $[29,30]$. Larger platelets are also a characteristic feature of ischemic stroke, detectable from the early hours of its onset; probably present well before the acute cerebrovascular event [31]. In the literature, only 1 study previously investigated platelet volume indices in IE [32]. However, the study was designed retrospectively in a small group of patients, and about one third of them had prosthetic valve endocarditis. None of our patients had prosthetic heart valves. We also excluded those being treated with platelet-inhibitors since a previous study reported reduced risk of symptomatic emboli associated with IE in patients who received continuous daily antiplatelet therapy before onset of IE [33].

\section{Limitations of the study}

A limitation of MPV as a marker of embolic risk is uncertainty as to whether the relationship between platelet size and the event risk is continuous or categorical. A broad spectrum of MPV values was seen in our analysis, ranging from 7.12 to $13.4 \mathrm{fL}$. Given the significant but still narrow absolute difference in mean values of MPV between embolic and non-embolic patients, the clinical utility of an isolated MPV in an individual's embolic risk is unclear. The MPV cut-off point that is most useful for predicting thromboembolic events in clinical practice remains unknown [10, $22]$. Another important consideration is the variability in the timing and methods of blood sample preparation and the type and calibration of particle counters, which may have had a significant impact in MPV measurements [8-10]. A previous study revealed that platelets swelled until $120 \mathrm{~min}$ in EDTA and until $60 \mathrm{~min}$ in citrate. There was a plateau of swelling after $120 \mathrm{~min}$ in EDTA and after $60 \mathrm{~min}$ in citrate. It was crucial to wait until stability of swelling had been reached. Therefore, they recommended that optimal measuring time should be $120 \mathrm{~min}$ after venipuncture if the tube containing EDTA was used [34]. In another report, there was an increase in MPV in EDTA within 30 min with a mean increase of $0.7 \mathrm{fL}$, corresponding to a $7.9 \%$ increase over baseline. MPV, however, continued to increase over the 24 -h period (overall $13.4 \%$ increase) with the majority of this increase by $6 \mathrm{~h}(11.2 \%)$ [35]. Recently 844 articles on the platelet volume indeces published between 1966 and 2011 have been reviewed [36]. In the vast majority of patients reviewed in this paper, blood samples were analyzed within $2 \mathrm{~h}$ of collection. All hematology analyzers had manufacturer-assigned 
ranges for platelet volume indices, which differed significantly between different analyzers depending on the technology used, but no external quality assessment schemes were currently available. Until such schemes were in place, the authors of this review recommended that blood samples for MPV should be analyzed within $2 \mathrm{~h}$ of venipuncture, in keeping with the methods used in most of the mentioned studies. For reliable MPV measurements, the potential influence of the anticoagulant must be carefully controlled by standardizing the time delay between sampling and analysis (less than $2 \mathrm{~h}$ ). In our study, in order to have a standardized time window for the analysis, blood samples of the subjects were collected in the same standard tubes containing EDTA and analyzed immediately within $60 \mathrm{~min}$ after venipuncture. Platelet activity seems to have a circadian variation in some reports [37], therefore all blood samples were drawn at 8.00 to 10.00 a.m. Lack of laboratory standardizations and cut-off values along with technical problems in platelet volume measurements presently limit the widespread usefulness of platelet volume indices.

Fragmentation of valvular vegetations as a result of turbulent blood flow within the cardiac chambers is supposed to lead to systemic emboli in IE. However, the results of several echocardiographic studies, which attempted to correlate the formation of an embolic event in IE with the morphological characteristics of a valvular vegetations, have been controversial $[6,7,38,39]$. Data are conflicting with regard to prognostic implications of vegetation characteristics such as shape, mobility and size for the occurrence of thromboembolic events. In our relatively large study population, although there was a trend toward a higher number of vegetations detected in embolic patients, this did not reach statistical significance. Similarly, location, mobility and attachment characteristics of vegetations were also identical in embolic and non-embolic groups. Not surprisingly, there was a significant correlation between larger vegetations and embolic events.

Interesting areas for future investigation in the field of IE are whether the reduction of MPV in the individual patient will lead to a change in thromboembolic outcome and whether a high-risk subgroup of patients with IE who had increased platelet activity reflected as elevated MPV warrants more aggressive treatment. It is likely that all therapies, including appropriate antimicrobial agents that lower mortality, also decrease thromboembolic complications. There is no information on the efficiency of heparin in IE; thus its use is not recommended because of the risk of hemorrhage, except for patients at perceived risk of pulmonary embolus or those with prosthetic heart valves. Platelet-inhibitors have been of interest as adjunctive therapy in IE because of the integral role of platelets in thromboembolic events of these patients. However, data on the use of platelet-inhibitors in IE patients to prevent major embolic events are insufficient $[40,41]$. Aspirin was found to exert little, if any, effect on MPV [42]. The failure to affect platelet size was also reported in the case of dual antiplatelet (aspirin + clopidogrel) therapy [43].

\section{Conclusions}

The present study demonstrated that IE patients with embolic events had increased MPV and PDW values, compared to non-embolics. Future prospective studies with standardized measurements may clarify the clinical role of platelet volume indices in thromboembolic complications of IE.

\section{Conflict of interest: None declared}

\section{References}

1. Netzer RO, Zollinger E, Seiler C, Cerny A. Infective endocarditis: Clinical spectrum, presentation and outcome: An analaysis of 212 cases 1980-1995. Heart, 2000; 84: 25-30.

2. Hart GH, Foster JW, Luther MF, Kanter MO. Stroke in infective endocarditis. Stroke, 1990; 21: 695-700.

3. Heiro M, Nikoskelainen J, Engblom E, Kotilainen E, Marttila R, Kotalainen P. Neurologic manifestations of infective endocarditis: A 17 year experience in a teaching hospital in Finland. Arch Intern Med, 2000; 160: 2781-2787.

4. Wallace SM, Walton BI, Kharbanda RK, Hardy R, Wilson AP, Swanton RH. Mortality from infective endocarditis: Clinical predictors of outcome. Heart, 2002; 88: 53-60.

5. Thyny F, Disalvo G, Belliard O et al. Risk of embolism and death in infective endocarditis: Prognostic value of echocardiography: A prospective multicenter study. Circulation, 2005; 112: 69-75.

6. Sanfilippo AJ, Picard MH, Newell JB. Echocardiographic assessment of patients with infective endocarditis; prediction of risk for complications. J Am Coll Cardiol, 1991; 18: 1191-1199 .

7. Habib G. Embolic risk in subacute bacterial endocarditis: Role of transesophageal echocariography. Curr Cardiol Rep, 2003; 5: 129-136.

8. Lance MD, Sloep M, Henskens YMC, Marcus MAE. Mean platelet volume as a diagnostic marker for cardiovascular disease: Drawbacks of preanalytical conditions and measuring techniques. Clin and Appl Thromb Hemost, 2012; 18: 561-568.

9. Leader A, Pereg D, Lishner M. Are platelet volume indeces of clinical use? A multi-disciplinary review. Ann Med, 2012; 44: 805-816.

10. Tsiara S, Elisaf M, Jagroop IA, Mikhailidis DP. Platelets as predictors of vascular risk: Is there a practical index of platelet activity? Clin Appl Thromb Hemost, 2003; 9: 177-190. 
11. Durack DT, Lukes AS, Bright DK. New criteria for diagnosis of infective endocarditis: Utilization of specific echocardiographic findings. Am J Med, 1994; 96: 200-209.

12. Shapiro SM, Young E, De Guzman S et al. Transesophageal echocardiography in the diagnosis of infective endocarditis. Chest, 1994; 105: 377-382.

13. Hill VL, Simpson VZ, Higgins JM et al. Evaluation of the performance of the Sysmex XT-2000i hematology analyzer with whole bloods stores at room temperature. Lab Med, 2009; 40: 709-718.

14. Kamath S, Blann AD, Lip GY. Platelet activation: Assessment and quantification. Eur Heart J, 2001; 22: 1561-1571.

15. Bath PM, Butterworth RJ. Platelet size: measurement, physiology and cascular disease. Blood Coagul Fibrinolysis, 1996; 7: 157-161.

16. Valtonen V, Kuikka A, Sytjanen J. Thromboembolic complications in bacteremic infections. Eur Heart J, 1993; 14 (suppl. K): 20-23.

17. Syrjanen J. Infection as a risk factor for cerebral infection. Eur Heart J, 1993; 14 (suppl. K): K17-K19.

18. De Meyer GRY, Herman AG. Vascular endothelial dysfunction. Progr Cardiovasc Dis, 1997; 39: 325-342.

19. Munro JM. Endothelial-leukocyte adhesive interections in flamatory disease. Eur Heart J, 1993; 14 (suppl. K): K72-K77.

20. Korkmaz S, Ileri M, Hisar I, Yetkin E, Koşar F. Increased levels of soluble adhesion molecules, E-selectin and P-selectin, in patients with infective endocarditis and embolic events. Eur Heart J, 2001; 22: 874-878.

21. Ileri M, Alper A, Senen K et al. Effect of infective endocarditis on blood coagulation and platelet activation and comparison of patients with to those without embolic events. Am J Cardiol, 2003; 91: 689-692.

22. Chu SG, Becker RC, Berger PB et al. Mean platelet volume as a predictor of cardiovascular risk: a systematic review and meta-analysis. J Thromb Haemost, 2010; 8: 148-156.

23. Machin SJ, Briggs C. Mean platelet volume: A quick and easy determinant of thrombotic risk. J Thromb Haemost, 2009; 8: 146-147.

24. Vizioli L, Muscari S, Muscari A. The relationship of mean platelet volume with the risk and prognosis of cardiovascular disease. Int J Clin Pract, 2009; 63: 1509-1515.

25. Gasparyan AY, Ayvazyan L, Dimitri P, Mikhailidis DP, George D, Kitas GD. Mean platelet volume: A link between thrombosis and inflamation. Curr Pharm Des, 2011; 17: 47-58.

26. Martin JF, Bath PM, Burr ML. Influence of platelet size on outcome after myocardial infarction. Lancet, 1991; 338: 1409-1411.

27. Pereg D, Berlin T, Mosseri M. Mean platelet volume on admission correlates with impaired response to thrombolysis in patients with ST-elevation myocardial infarction. Platelets, 2010; 21: 117-121.

28. Huczek Z, Kochman J, Filipiak KJ et al. Mean platelet volume on admission predicts impaired reperfusion and long-term mortality in acute myocardial infarction treated with percutaneous coronary intervention. J Am Coll Cardiol, 2005; 46: 284-290.

29. Maden O, Kacmaz F, Selcuk H et al. Relationship of admission hematological indices with myocardial reperfusion abnormalities in acute ST segment elevation myocardial infarction patients treated with primary percutaneous coronary interventions. Can J Cardiol, 2009; 25: e164-e168.

30. Yang A, Pizzulli L, Lüderitz B. Mean platelet volume as a market of restenosis after percutaneous transluminal coronary angioplasty in patients with stable and unstable angina pectoris. Thromb Res, 2006; 117: 371-377.

31. O'Malley T, Langhorne P, Elton RA, Stewart C. Platelet size in stroke patients. Stroke, 1995; 26: 995-999.

32. Günebakmaz O, Kaya MG, Kaya EG et al. Mean platelet volume predicts embolic complications and prognosis in infective endocarditis. Int J Infect Dis, 2010; 14: e982-e985.

33. Anavekar NS, Tleyjeh IM, Anavekar NS et al. Impact of prior antiplatelet therapy on the risk of embolism in infective endocarditis. Clin Infect Dis, 2007; 44: 1180-1186.

34. Lancé MD, van Oerle R, Henskens YM, Marcus MA. Do we need time adjusted mean platelet volume measurements? Lab Hematol, 2010: 16: 28-31.

35. Bowles KM, Cooke LJ, Richards EM, Baglin TO. Platelet size has diagnostic predictive value in patients with thrombocytopenia. Clin Lab Haem, 2005; 27: 370-373.

36. Leader A, Pereg D, Lishner M. Are platelet volume indeces of clinical use? A multidisciplinary review. Ann Med, 2012; 44: 805-816.

37. Dalby MC, Davidson SJ, Burman JF, Davies SW. Diurnal variation in platelet aggregation with the PFA-100 platelet function analyzer. Platelets, 2000; 11: 320.

38. Steckelberg JM, Murphy JG, Ballard D. Emboli in infective endocarditis: The prognostic value of echocardiography. Ann Intern Med, 1991; 114:635-640.

39. Buda AJ, Zotz RJ, Le Mire MS, Bach DS. Prognostic significance of vegetations detected by two-dimensional echocardiography in infective endocarditis. Am Heart J, 1986; 112: 1291-1296.

40. Chan KL, Dumesnil JG, Cujec B et al. A randomized trial of aspirin on the risk of embolic events in patients with infective endocarditis. J Am Coll Cardiol, 2003; 42: 775-780.

41. Baddour LM, Wilson WR, Bayer AS et al. Committee on Rheumatic Fever, Endocarditis, and Kawasaki Disease; Council on Cardiovascular Disease in the Young; Councils on Clinical Cardiology, Stroke, and Cardiovascular Surgery and Anesthesia; American Heart Association; Infectious Diseases Society of America. Infective endocarditis: Diagnosis, antimicrobial therapy, and management of complications: A statement for healthcare professionals from the Committee on Rheumatic Fever, Endocarditis, and Kawasaki Disease, Council on Cardiovascular Disease in the Young, and the Councils on Clinical Cardiology, Stroke, and Cardiovascular Surgery and Anesthesia, American Heart Association: Endorsed by the Infectious Diseases Society of America. Circulation, 2005; 111: e394-e434.

42. Erhart S, Beer JH, Reinhart WH. Influence of aspirin on platelet count and volume in humans. Acta Haematol, 1999; 101: 140-144.

43. Guthikonda S, Alviar CL, Vaduganathan $\mathrm{M}$ et al. Role of reticulated platelets and platelet size heterogeneity on platelet activity after dual antiplatelet therapy with aspirin and clopidogrel in patients with stable coronary artery disease. J Am Coll Cardiol, 2008; 52: 743-749. 